Objectives A pilot study to assess the effect of increased air pressure in a hyperbaric chamber on patients with active rheumatoid arthritis.

Methods Seven out-patients with active rheumatoid arthritis (RA) and taking at least one disease modifying anti-rheumatic drug (DMARD) entered a hyperbaric chamber for $30 \mathrm{~min}$. Patients were randomised to control and test groups and assessed in a double-blind fashion. The test group had the atmospheric pressure increased to 2 atmospheres. The control group did not have the pressure altered. Active disease was defined as a pain score on a $100 \mathrm{~mm}$ visual analogue scale (VAS) greater than 60 $\mathrm{mm}$ and 3 out of 4 from the following: - ESR $>28 \mathrm{~mm} / \mathrm{hr},>6$ swollen joints, $>6$ tender joints and early morning stiffness $>45$ min. The following were recorded or measured: pain score (by $100 \mathrm{~mm}$ VAS), number of swollen joints, number of tender joints, patients global score on a $100 \mathrm{~mm}$ VAS, ESR and CRP. The above measurements were repeated $30 \mathrm{~min}$ after leaving the hyperbaric chamber, the following day and 7 days later. Disease Activity Scores (DAS) were calculated using the DAS 28.

Results Four female and three male patients participated. Mean age was 58.4 years (range:41-71 yrs), mean disease duration 10.86 yrs $(4-20$ yrs). In the test group the pain score of 1 patient was reduced by $60 \%$ and DAS by $38 \%$ at 24 hrs but had returned to the pre-test values by 7 days. Pain scores from the other 2 patients did not alter. In the control group the pain scores of 2 patients had fallen by $60 \%$ and $64 \%$ and at 30 mins post-test. This effect was maintained to 7 days for the latter patient whose DAS fell by $54 \%$ and at $24 \mathrm{hrs}$ for the former patient whose DAS did not change, however, this patient's oral steroid dose was inadvertently altered between the $24 \mathrm{hr}$ and 7 day assessment. All patients in the test group had reduced swollen joint counts at 30 mins and 24 hrs post-test, this was maintained at 7 days in 2 patients; the tender joint count was reduced in 1 patient at 24 hrs only. 3 out of the 4 control patients had reduced swollen joint counts from 30 mins post-test this was maintained at $24 \mathrm{hrs}$ and 7 days, the same patients also had reduced tender joint counts. There was no change in the ESRs in either group. The CRP in 1 patient from the test group fell at $24 \mathrm{hrs}$ and further decreased at 7 days. In the control group CRP decrease in 1 patient at 24 hrs only, neither of these 2 patients had reduced pain scores or DAS during the study.

Conclusion In the small number of patients studied, increased atmospheric pressure does not appear to have any effect on active rheumatoid arthritis. There also appears to be a large placebo response.

\section{THU0140 RHEUMATOID ARTHRITIS (RA) IN VILNIUS AND OSLO: A COMPARISON REGARDING DISEASE AND HEALTH MEASURES}

${ }^{1} \mathrm{JH}$ Dadoniene, ${ }^{2} \mathrm{~T}$ Uhlig, ${ }^{1} \mathrm{~S}$ Stropuviene, ${ }^{1} \mathrm{~A}$ Venalis, ${ }^{3} \mathrm{~A}$ Boonen, ${ }^{2} \mathrm{TK}$ Kvien. ${ }^{1} \mathrm{The}$ Clinic of Internal Medicine and Rheumatology, Vilnius University, Vilnius, Lithuania; 'Department of Rheumatology, Diakonhjemmet Hospital, Oslo, Norway; ${ }^{3}$ Department of Rheumatology, University Hospital, Maastricht, Netherlands

\subsection{6/annrheumdis-2001.1042}

Background In Bone and Joint Decade comparative studies between countries could contribute to better understanding of disease burden of rheumatic diseases.

Objectives To compare disease and health status measures in RA patient cohorts from Vilnius, Lithuania and Oslo, Norway.

Methods For 201 patients from Vilnius RA register, matched cases according to age (mean 55.9 (SD1.0)) and gender (167 female) were identified in Oslo RA register. Study variables included sociodemographic characteristics, health status measures (MHAQ, SF-36, pain VAS), disease process measures (disease activity score (DAS-28)), ESR) and previous/current treatment.

Results Most disease activity and health status measures were worse in Vilnius patients (Table 1). Rheumatoid factor positivity was observed in 61 vs.49\% $(\mathrm{p}=0.03)$ in Vilnius and Oslo, respectively. Methotrexate was currently or previously used by 36 vs. $49 \%$ ( $\mathrm{p}=0.05)$ and azatioprine by 29 vs. $13 \%$ ( $\mathrm{p}=$ 0.000 ) in Vilnius and Oslo. For other DMARDS, including corticosteroids no important differences were observed.

\begin{tabular}{lll} 
Abstract THU0140 Table 1 & \\
\hline & Vilnius $(\mathbf{n}=\mathbf{2 0 1})$ & 0slo $(\mathbf{n}=\mathbf{2 0 1})$ \\
\hline MHAQ (1-4) & $2.30(0.78)$ & $1.63(0.53)^{*}$ \\
Pain (0-100 VAS) & $59.4(20.0)$ & $36.2(22.1)^{*}$ \\
Patient global (1-5) & $4.9(1.8)$ & $4.2(2.0)^{*}$ \\
Investigator global (0-100) & $47.6(22.8)$ & $29.2(24.7)^{*}$ \\
DAS-28 & $5.26(1.0)$ & $4.37(1.4)^{*}$ \\
SF-36, physical phunctioning & $35.2(25.2)$ & $48.5(25.6)^{*}$ \\
SF-36, mental health & $54.6(21.0)$ & $68.7(19.7)^{*}$ \\
ESR mm/hr & $28.6(13)$ & $20.5(17.5)^{*}$ \\
\hline *-p-value $<0.001, t$-test for continuos values and chi-2 for counts.
\end{tabular}

Conclusion In this two-country comparison RA patients from Lithuania had generally worse health status and higher disease activity. This difference could represent geographic differences in disease severity or unequal access to rheumatology care.

\section{THU0141 THE DIAGNOSTIC VALUE OF RHEUMATOID FACTORS AND ANTIBODIES TO CYCLIC CITRULLINATED PEPTIDE (CCP) IN EARLY ARTHRITIS}

${ }^{1}$ LM Jansen, ${ }^{2}$ IE Van der Horst-Bruinsma, ${ }^{1} D$ Van Schaardenburg, , $R J$ Van de Stadt, ${ }^{1} \mathrm{MH}$ De Koning, ${ }^{2}$ BA Dijkmans. 'Department of Rheumatology, Jan Van Breemen Instituut, Amsterdam; ${ }^{2}$ Department of Rheumatology, VU Medical Center, Amsterdam, The Netherlands

10.1136/annrheumdis-2001.1043

\section{Background}

Objectives To study the diagnostic value of IgM-RF, IgA-RF, anti-CCP and combinations of these antibodies measured at baseline to discriminate rheumatoid arthritis (RA) from undifferentiated polyarthritis (UPA) in patients with recent onset arthritis.

Methods Early arthritis patients with peripheral arthritis of 2 or more joints and less than 3 years of symptom duration were clinically diagnosed as RA or UPA by the rheumatologist during the first year. Excluded from the study were patients with bacterial, psoriatic, crystal-induced arthritis or spondylarthropathy. Optimal cut-off values for serum IgM-RF, IgA-RF and anti-CCP were deduced from Receiver Operating Characteristics (ROC) curves. Results 379 patients (69\% female, median age 57 (17-86)) were included. 258 patients were clinically diagnosed as RA and 121 as UPA. The Table 1 shows optimal cut-off values for IgM-RF (IU/ml), IgA-RF (arbitrary units: AU/ml) and anti-CCP (AU/ml) with the matching specificity and sensitivity percentages.

Both the IgM-RF $>40$ and anti-CCP $>50$ have a high specificity but the sensitivities are low. Because in many RA patients the occurrence of IgM-RF and anti-CCP antibodies are independent from each other, the best test criterion proved to be the 